\title{
Effects of acceptor doping on a metalorganic switch: DFT vs. model analysis
}

\author{
T. Ślusarski, ${ }^{a}$ T. Kostyrko, ${ }^{a}$ V. M. Garcia-Suarez ${ }^{b, c}$ \\ (a) Faculty of Physics, A. Mickiewicz University, ul. Umultowska 85, 61-614 Poznań, Poland \\ (b) Departamento de Física, Universidad de Oviedo, 33007 Oviedo, Spain \\ (c) Nanomaterials and Nanotechnology Research Center (CINN), Oviedo, Spain
}

(Dated: October 20, 2017, version 13)

\begin{abstract}
We propose a molecular switch based on copper dioxolene molecules with valence tautomeric properties (spin-crossover). We study the system with first-principles methods and a model Hamiltonian that can properly account for electronic correlations in these complex spin-crossover molecular systems. We compute the transmission of a junction with a $\mathrm{Cu}$-dioxolene unit sandwiched between gold electrodes and show that it depends on the spin state of the molecule. We also study effects of doping with $\mathrm{ICl}_{2}$ acceptor molecules on the magnetic and electronic features of the device. We find that in the absence of dopants the $\mathrm{Cu}$-dioxolene unit is weakly charged in a $S=1 / 2$ spin state. However, the acceptors increase the charge state of molecule and make possible a transition between the high-spin $(S=1)$ triplet and the low-spin $(S=0)$ singlet. The $I-V$ dependence shows a manifestation of spin filtering and a multistable behavior that can have several applications in nanoscale electronic devices.
\end{abstract}

\section{INTRODUCTION}

Studies of electronic transport through the molecular junctions including magnetic molecules remain an important part of activity in the field of molecular electronics. The ability to control the spin degrees of freedom with external magnetic and electric fields enhances the flexibility of applications of these molecules in devices such as as switches, molecular sensors, memories, and spin filters ${ }^{1-3}$. A special role in this research is played by magnetic molecules exhibiting transitions between various magnetic spin states, i.e. spin-crossover ( $\mathrm{SCO}$ ) molecules. ${ }^{4-7}$ In these molecules, the change of spin is often accompanied by a change of conformation and a redistribution of the electronic charge within the molecule. When this leads to a change of the formal valence of the magnetic ion, the phenomenon is called a valence tautomerism. In bulk systems, such transformations can be induced by temperature ${ }^{8,9}$ or pressure ${ }^{10,11}$, as well as by light ${ }^{12,13}$ or even by the adsorption of molecules ${ }^{14-16}$. In such crystals or other systems, such as thin films, the electronic transport properties depend substantially on the spin state of the magnetic ion ${ }^{9,17,18}$ and could therefore be employed in molecular junctions for switching functionalities. Incorporating SCO molecules in molecular junctions is facilitated by their structure, which typically includes aromatic ligands that may be effectively functionalized with organic linkers that allow them to bind to metal leads while preserving the SCO functionality ${ }^{19}$.

A detailed understanding of the mechanisms of transport in junctions including SCO molecules needs the application of theoretical approaches that reliably describe the multiorbital electronic structure of these complex metalorganic molecules. Moreover, these approaches should accurately describe the magnetic transformation and properly account for correlation effects. SCO systems still pose a challenge for first principles methods, which are commonly based on density functional theory (DFT) combined with the nonequilibrium Green functions approach (NEGF). The main problem is that standard local exchange correlation (XC) functionals, like LDA or GGA, may be questionable in these systems because of the importance of correlations on the magnetic centers. The use of more accurate methods of quantum chemistry is impeded by the size of the computational unit cell, which has to incorporate also a large portion of the leads. Therefore, many studies performed so far on these systems ${ }^{20-26}$ included some improvements of standard functionals to better treat exchange or reduce self-interaction effects inherent to local XC functionals. These extensions are usually based on hybrid XC functionals like B3LYP ${ }^{27,28}$, a form of SIC (self interaction correction) ${ }^{29}$ or LDA+U type approaches ${ }^{30,31}$.

However, the mentioned approaches are still based on the one particle eigenstates of the Kohn-Sham Hamiltonian of DFT. Although they can accurately describe the influence of correlation effects on the total energy and the electron density of the system, the effects of electron interactions on the electronic structure are still of mean-field type. Therefore, it is of considerable importance to develop methods that can properly account for effects of electron correlations and treat in a realistic manner the complexity of molecular structures in the SCO systems. A solution may be to use first principles calculations to derive effective many body Hamiltonians that can be subsequently used to study the molecular system with some controllable approximations.

The purpose of the present paper is to study theoretically a model metalorganic system that exhibits properties of a SCO system. Such model is a simplified representation of a $\mathrm{Cu}$-dioxolene molecule and can also describe a large group of systems exhibiting valence tautomerism. We first study the transport properties of the molecular system sandwiched between gold electrodes with a combination of DFT with the GGA+U parametrization and NEGF. We also investigate the possibility of controlling the magnetic and transport properties of the molecule by doping the system 
with acceptor molecules. We show that the molecule can work as a spin filter and, in some voltage regions, may also exhibit a multistable behavior. Next, we quantitatively reproduce the main features of the transport characteristics of the system with a model Hamiltonian. The advantage of such model Hamiltonian is twofold. First, it is very useful for understanding details of the results of the DFT computations. Second, its simple form is amenable to improving the treatment of correlation effects in the transport properties.

\section{MODEL OF JUNCTION AND COMPUTATIONAL METHOD}

We consider a molecular junction with a Cu-dioxolene complex bound to two gold (111) surfaces with butanethiol linkers (see figure 1). The butanethiol chain is connected with its end carbon atom to the benzene ring of the dioxolene part. After optimization, the sulfur head of the linker is placed on the $\mathrm{Au}(111)$ surface near the bridge position.

The molecular junction was simulated in a periodic unit cell. The (111) surface on each lead was made of two $5 \times 5$ atom layers of $\mathrm{Au}$ atoms. The leads, right after the surface layers, were constructed as 3 layers of $5 \times 5$ atoms. Each electrode had then 5 layers of gold atoms. We set the distance between the surfaces to match the values of interatomic distances between atoms in a relaxed symmetric structure of the molecule adsorbed on the surface with one butanethiol chain. ${ }^{32}$ Then, the junction was optimized by keeping the leads frozen and allowing the atoms at the surface region to relax.

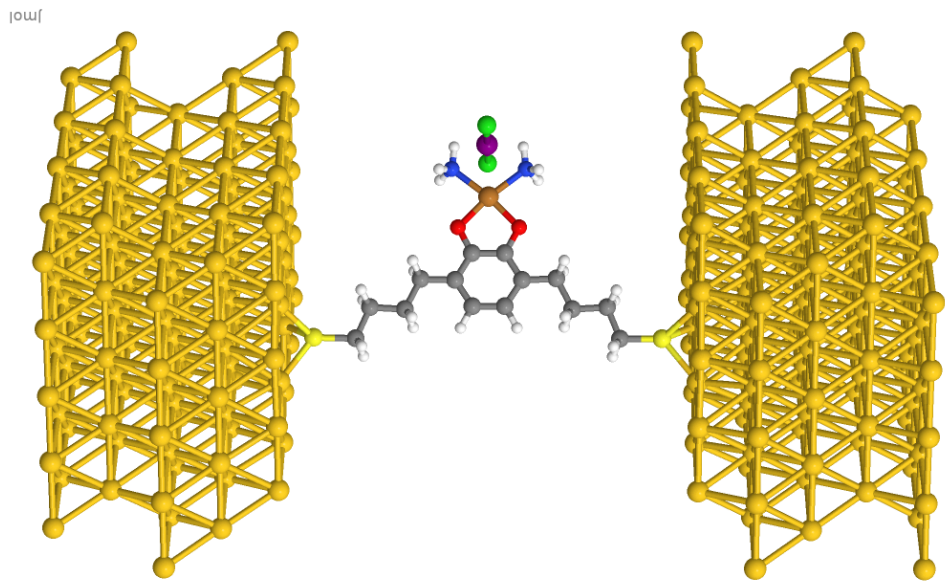

FIG. 1: (color online) Structure of the molecular junction: metalorganic molecule linked through butanethiol chains to the $\mathrm{Au}(111)$ surfaces. This structure corresponds to the planar symmetric state with the neighboring acceptor ion. ${ }^{33}$.

We considered also the effects of doping with acceptor ions $\mathrm{ICl}_{2}$. The ion was placed roughly at the center of the unit cell in order to preserve overall symmetry of the computational unit cell. The acceptor ion could be orientated parallel or perpendicularly with respect to the surface. In our simulations, the ion typically accepts around $0.5|e|$ as determined from the Mulliken population analysis. The computations were performed using the DFT method implemented in the SIESTA package ${ }^{34}$. The advantage of this code in simulating electronic transport in nanostructures comes from the fact that it uses finite range basis function and implements pseudopotentials to reduce the number of the electronic degrees of freedom. The electronic densities in an applied voltage are computed using nonequilibrium Keldysh-type Green's functions.

In the simulations we used the generalized gradient approximation (GGA) with the Perdew-Burke-Erzenhof (PBE) parametrization $^{35}$ extended with a Hubbard-type term treated in the HF approximation $\left(\mathrm{GGA}+\mathrm{U}\right.$ method $\left.^{30,31}\right)$. This functional takes the following form:

$$
\begin{array}{r}
E_{\mathrm{GGA}+\mathrm{U}}[\rho(\vec{r})]=E_{\mathrm{GGA}}[\rho(\vec{r})]+ \\
E_{\text {Hubbard }}\left[\left\{n^{\sigma}\right\}\right]-E_{\mathrm{DC}}\left[\left\{n^{\sigma}\right\}\right]
\end{array}
$$

where $\rho(\vec{r})$ is the electron charge density and $\left\{n^{\sigma}\right\}$ is the reduced density matrix for $d$-electrons, $E_{\mathrm{GGA}}$ is the GGA functional, $E_{\text {Hubbard }}$ denotes the Hubbard-like functional, and $E_{\mathrm{DC}}$ is the double-counting correction, which eliminates the contribution from the $d$ electrons already included in $E_{\text {GGA }}$. We assumed $U=6 \mathrm{eV}$ for all computations in this work. This value was suggested from studies of copper complexes, as giving reliable estimates of effective exchange couplings and a good agreement with the B3LYP hybrid exchange-correlation potential. ${ }^{36}$ 
TABLE I: Net and selected Mulliken atomic populations in units of charge $(|e|)$ and spin $\left(\mu_{B}\right)$ of the Cu-dioxolene complex linked to the $\mathrm{Au}(111)$ surfaces with butanethiol linkers. Both symmetric HS and twisted LS structures are shown. The abbreviation acc means the presence of the acceptor ion in the unit cell. $\mathrm{T}_{\uparrow}\left(\mathrm{T}_{\downarrow}\right)$ is the value of the transmission function at Fermi level for spin up (down).

\begin{tabular}{lccccccc}
\hline \hline & $\mathrm{Q}_{\mathrm{Tot}}$ & $\mathrm{M}_{\mathrm{Tot}}$ & \multicolumn{1}{c}{$\mathrm{Q}_{\mathrm{Cu}}$} & \multicolumn{1}{c}{$\mathrm{M}_{\mathrm{Cu}}$} & \multicolumn{1}{c}{$\mathrm{M}_{\mathrm{O}}$} & $\mathrm{T}_{\uparrow} \times 10^{6}$ & $\mathrm{~T}_{\downarrow} \times 10^{6}$ \\
\hline HS & 0.371 & 1.265 & 0.545 & 0.555 & 0.211 & 0.371 & 1.78 \\
LS & 0.279 & 0.803 & 0.416 & 0.224 & 0.151 & 1.24 & 0.921 \\
HSacc & 0.715 & 1.663 & 0.601 & 0.589 & 0.259 & 0.405 & 2.59 \\
LSacc & 0.685 & 0.389 & 0.601 & 0.573 & -0.025 & 3.7 & 0.85 \\
\hline \hline
\end{tabular}

We used in all calculations Troullier-Martins norm-conserving pseudopotentials ${ }^{37}$ with scalar relativistic corrections and nonlinear core corrections (these last ones, for every atom but hydrogen). We optimized the pseudopotentials with the simplex and swarm algorithm within the SIESTA package in order to find a reasonable compromise between transferability and softness.

The pseudoatomic basis set was constructed using soft confining potentials with an energy of 50 Ry and an inner radius of 0.7 Bohr. We used a double-zeta polarized (DZP) basis with a splitting norm of 0.5 for the hydrogen atom and 0.15 otherwise. The value of the PAO.EnergyShift, parameter that determines the range of the basis function, was set to 0.005 Ry to create sufficiently long-ranged pseudoatomic orbitals. The basis of the acceptor ion was further extended by including diffuse orbitals (unoccupied long range orbitals of higher quantum number than the valence orbitals). The range of the diffuse orbitals that we chose allows overlaps only with the molecular subunit, leading to substantial transfer of charge from it. The MeshCutoff parameter was set equal to 600 Ry and the GridCellSampling option was used to avoid differences coming from the shape of the unit cell. The electronic temperature in the Fermi-Dirac distribution function was set to $25 \mathrm{meV}$. The self-consistent procedure was run until the density matrix and Hamiltonian differences were smaller than $10^{-4}$. The structures were relaxed in the equilibrium simulations with a conjugate gradient algorithm until all forces were smaller than $0.04 \mathrm{eV} / \AA$. The relaxed structures were taken as an frozen input for next NEGF simulations.

The spin-dependent electronic current was computed using the Landauer-Büttiker formula:

$$
I_{\sigma}(V)=\frac{e}{h} \int d E T_{\sigma}(E, V)\left(f_{L}-f_{R}\right)
$$

where $f_{L}, f_{R}$ are the Fermi factors for the left and right lead, respectively, and the transmission function $T_{\sigma}(E, V)$ for spin $\sigma$ is computed using the NEGF method implemented in SIESTA. In order to reduce substantially the numerical problem, related to the large size of the computational unit cell, we reduced the basis of the gold leads to a SZP basis. All other atoms (including the atoms of the surface layers) were described using the DZP basis. In order to confirm the reliability of this procedure, we used the GOLLUM code ${ }^{38}$ to compare results obtained with reduced leads to results obtained with a DZP basis in all atoms.

\section{RESULTS OF THE DFT ANALYSIS}

\section{A. Equilibrium case}

We obtained two relaxed structures: a high-spin conformation (HS) with preserved planar symmetry (perpendicular to the Au surface) and a low-spin conformation without any symmetry constraints (LS). The LS solution was lower in energy than the HS solution by about $0.3 \mathrm{eV}$.

Due to the linking to the leads with well insulating alkanethiols the molecule remains in the neutral charge state rather than in the $+|e|$ state of the isolated molecule (in the gas phase). The total charge of the molecular subunit is higher in the HS solution $(0.371|e|)$ than in the LS solution $(0.279|e|)$ (table I). The total magnetic moment has a value $1.265 \mu_{B}$ and $0.803 \mu_{B}$, for the HS and LS configurations, respectively. In the HS solution, the main contribution comes from the copper atom $\left(0.555 \mu_{B}\right)$ and from both oxygen atoms $\left(0.21 \mu_{B}\right.$ each). In the LS solution, apart from the copper atom $\left(0.224 \mu_{B}\right)$ and both oxygen atoms $\left(0.151 \mu_{B}\right.$ each), there is a slightly higher contribution coming from all carbon atoms in the benzene ring. The differences are visible in the spin density isosurfaces plots (figure 2). In the twisted LS solution, due to the mixing of the atomic orbitals, the spin is distributed more diversely than in the case of the symmetric HS solution, where the spin directly originates from the atomic spinorbitals. There is no spin 
transfer across the linkers and the surfaces remain diamagnetic. The local charge of the copper atom is different for both conformations, suggesting that these solutions may refer to different tautomers.

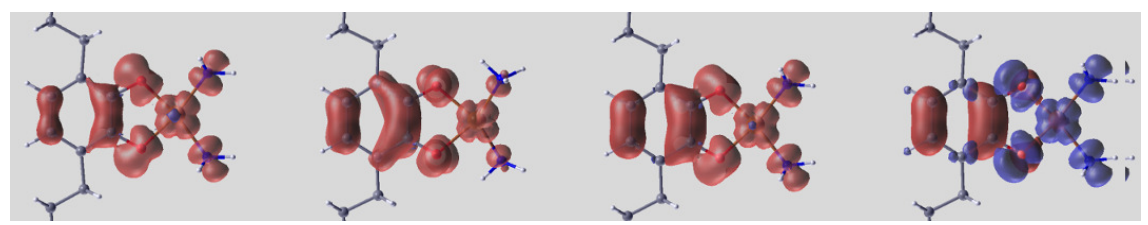

FIG. 2: (color online) Spin densities of $\mathrm{HS}, \mathrm{LS}, \mathrm{HS}+\mathrm{ICl}_{2}$, and $\mathrm{LS}+\mathrm{ICl}_{2}$ (from left to right ). The value chosen to plot the isosurfaces is $0.005^{39}$.

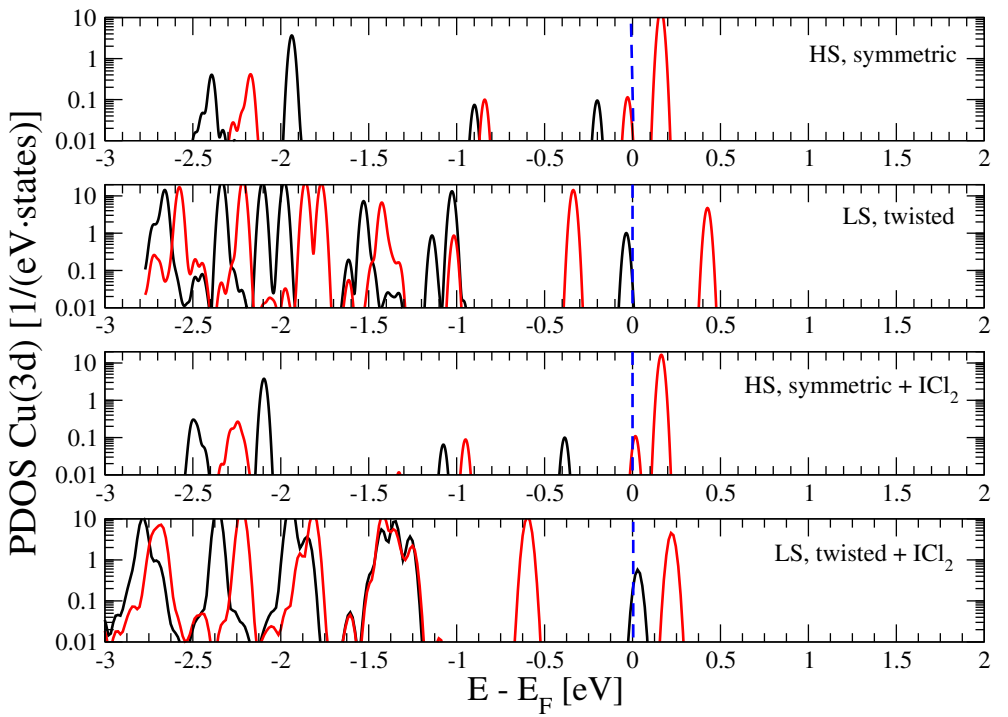

FIG. 3: (color online) Projected densities of states on the 3d orbitals of the copper atom.

In figure 3 we plot the projected densities of states (PDOS) on the $3 \mathrm{~d}$ orbitals of the copper atom for different spin solutions and structural configurations. In both solutions (HS and LS) we find a pinning of the HOMO to the Fermi level generated by charge transfer from the molecule to the leads. The antibonding LUMO is the most localized molecular orbital on the $3 \mathrm{~d}$ state. The HOMO-LUMO gap is bigger in the case of the twisted conformation (0.5 eV) than in the symmetric one $(0.1 \mathrm{eV})$.

We have plotted the local DOS for energy ranges around some of the most relevant peaks (HOMO-2, HOMO and LUMO) (figure 4) and found that these states resemble spinorbitals of the isolated molecule ${ }^{32}$. Note that these three orbitals belong to different symmetry classes of the point group of the isolated molecule. The HOMO and HOMO-1 are mainly localized on the p orbitals of the benzene ring. These will be most crucial in electronic transport under an applied voltage. The LUMO consist mainly of oxygen, nitrogen and copper atomic contributions, but has no significant contribution from the benzene ring. However, it extends to the sulfur atoms on the surface, leading to the conclusion that holes located on the molecular subunit with their center on the transition metal ion could strongly determine the current. This state has an occupied, bonding counterpart, seen as a peak located around $-1 \mathrm{eV}$ in the PDOS (fig 3). Since this pair of states is mainly contributing to the total magnetization, the tuning of the LUMO level with external magnetic or electric ${ }^{32}$ fields could change the transport properties of the junction.

In figure 5 we plot the transmission functions for both spin configurations in equilibrium. Since our results were obtained using leads with a reduced orbital basis, we first compared the SIESTA computations with the ones using the full DZP basis and computed with the GOLLUM package. We found a strong similarity of both transmission functions, and, most importantly, of the energy positions of the coherent peaks which are crucial to the resonant tunneling. We conclude that the reducing the orbital basis in the leads does not lead to significant changes of other physical quantities in the scattering region, such as the local occupations.

The sharp peaks in the transmission function correspond well to the peaks in the PDOS function projected on the copper atom (see figure 3). For the symmetric solution, the HOMO and LUMO levels are in the same spin phase. The transmission function at the Fermi level is clearly different for both spins (the values of the transmission are listed in 


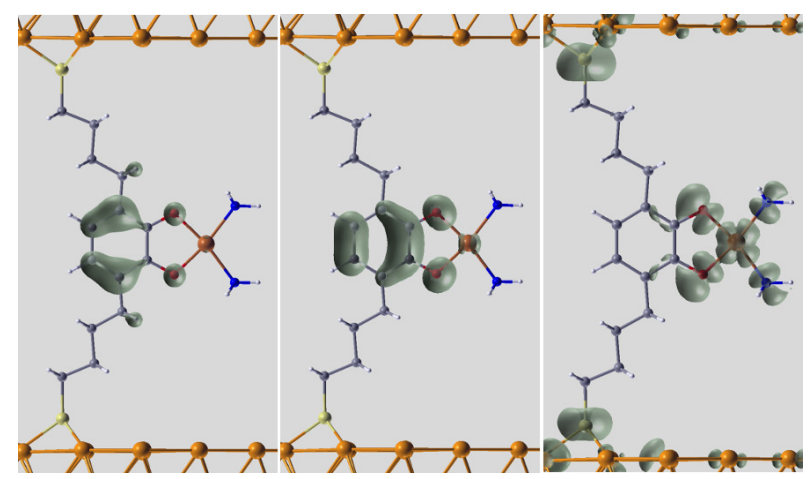

FIG. 4: (color online) Local densities of states for the HOMO-2, HOMO and LUMO levels of the symmetric HS solution.

table I). For the twisted solution, the HOMO level has an opposite spin to the LUMO level, which has moved higher in energy due to the twisting of the geometry of the molecule during the structural relaxation. The transmission function at the Fermi level is similar for both spins.

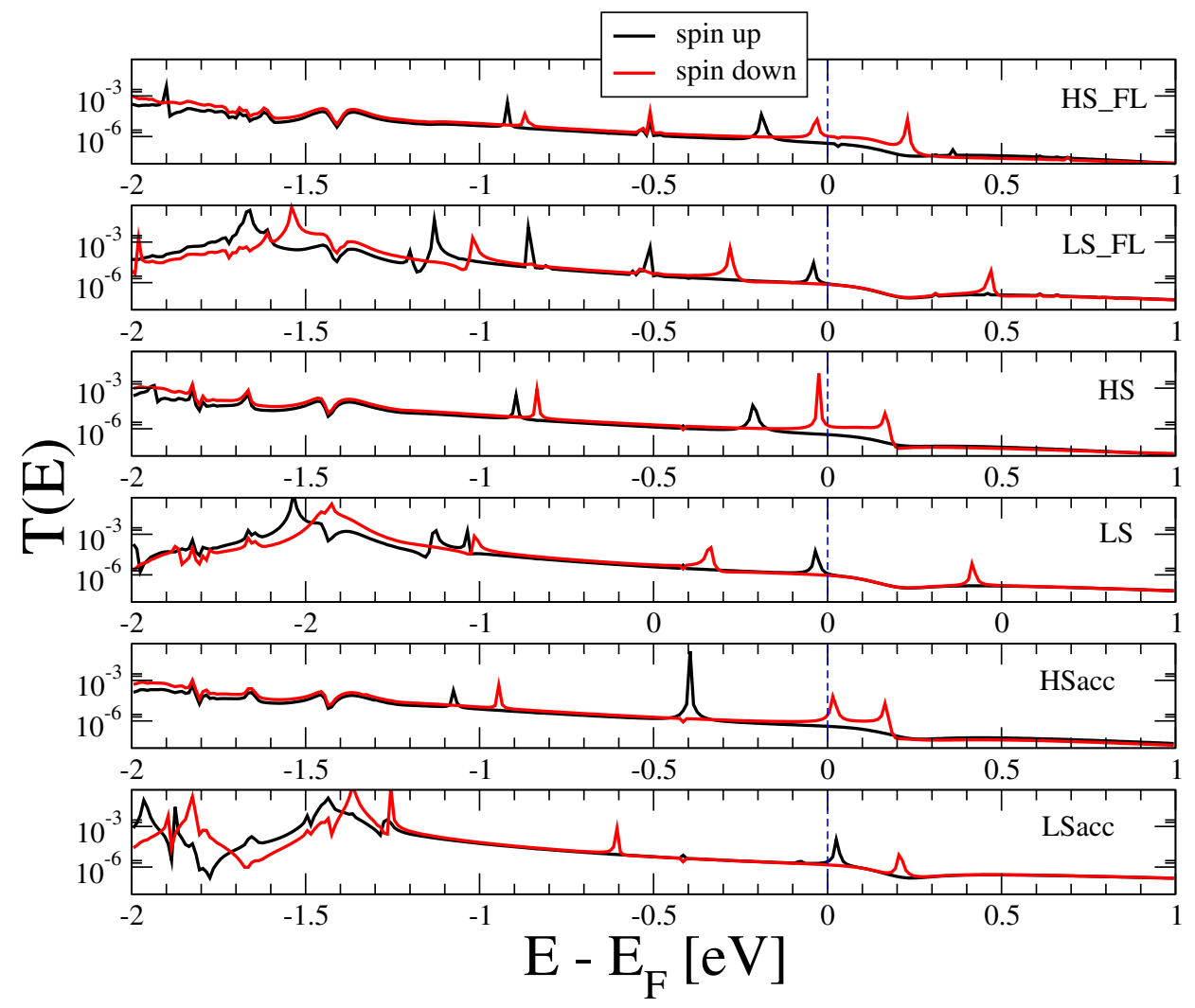

FIG. 5: (color online) Equilibrium transmission functions for some chosen conformations. FL corresponds to the use of full leads with DZP basis.

Analogously, we have analyzed two representative relaxed structures for the junction doped with acceptor molecules: the high-spin conformation with preserved planar symmetry (HSacc) and the low-spin twisted conformation (LSacc). The LS conformation has lower energy than the HS conformation by about $0.6 \mathrm{eV}$. Inclusion of the acceptors causes additional charging of the molecular subunit, which is effectively similar to the case when the adsorbed molecule is located in an external electric field ${ }^{32}$. In the HS solution, the total charge of the molecular subunit is similar to that of the LS solution $(0.715|e|)$ and $0.685|e|)$, respectively (table I). The total magnetic moment has a value $1.663 \mu_{B}$ and $0.389 \mu_{B}$, respectively. The molecular subunit in this case resembles more the isolated molecule (charged $+|e|$ ) rather than a neutral molecule with no doping. In the HS solution, the local magnetic moment on the copper atom 
$\left(0.589 \mu_{B}\right)$ is similar to the value in the LS solution $\left(0.573 \mu_{B}\right)$.

In the HS solution, the local magnetic moment on each oxygen atoms is equal to $0.26 \mu_{B}$. In the LS solution, however, it almost vanishes $\left(-0.025 \mu_{B}\right.$ each) and contributes with a different spin sign. The distribution of phase and spin is also different for both conformations, as seen in the spin density plots (figure 2). The effect of charging the molecule in the junction is observed as changes in the transmission functions (figure 5). In both solutions with doping, the HOMO level crossed the Fermi level and becomes unoccupied. Adding the acceptors causes the LUMO peak to move down in energy and come closer to the HOMO peak that crossed the Fermi level. The total values of the transmission functions at the Fermi level are similar in both solutions, but the major contribution comes from different spin.

\section{B. Nonequilibrium case}

The behavior of the system in the nonequilibrium case can be understood by analyzing the dependence of the transmissions peaks with the voltage. Each time a transmission peak enters or leaves the voltage window, the electron occupation of the corresponding molecular level undergoes a change, modifying other system characteristics. The voltage dependence of the transmission peaks is presented in figure 6 for the junction with the molecule in the HS and LS states, both with and without the acceptors.

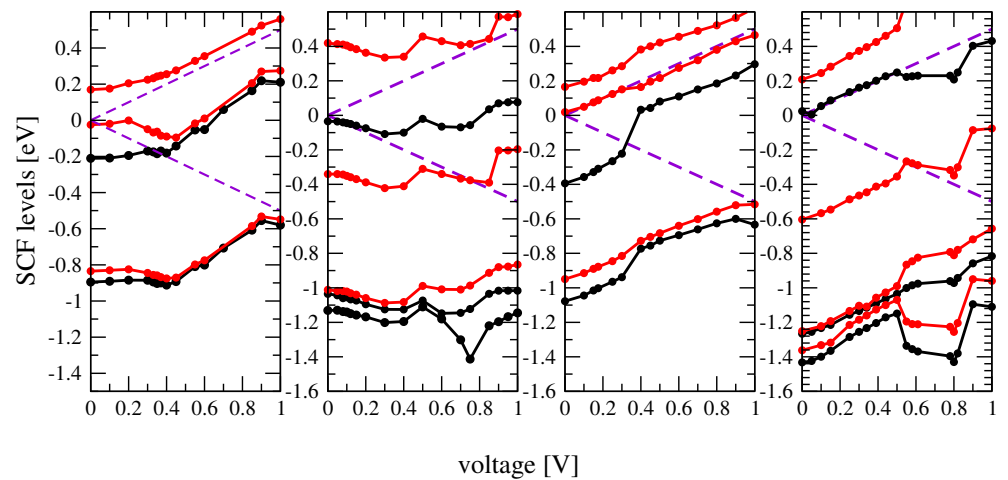

FIG. 6: (color online) Position of the peaks in the transmission functions with respect to the applied voltage. Black lines correspond to spin up and red lines to spin down polarization. The panels from left to right show results for junctions with a symmetric molecule, a twisted molecule, a symmetric molecule with acceptors and a twisted molecule with acceptors.

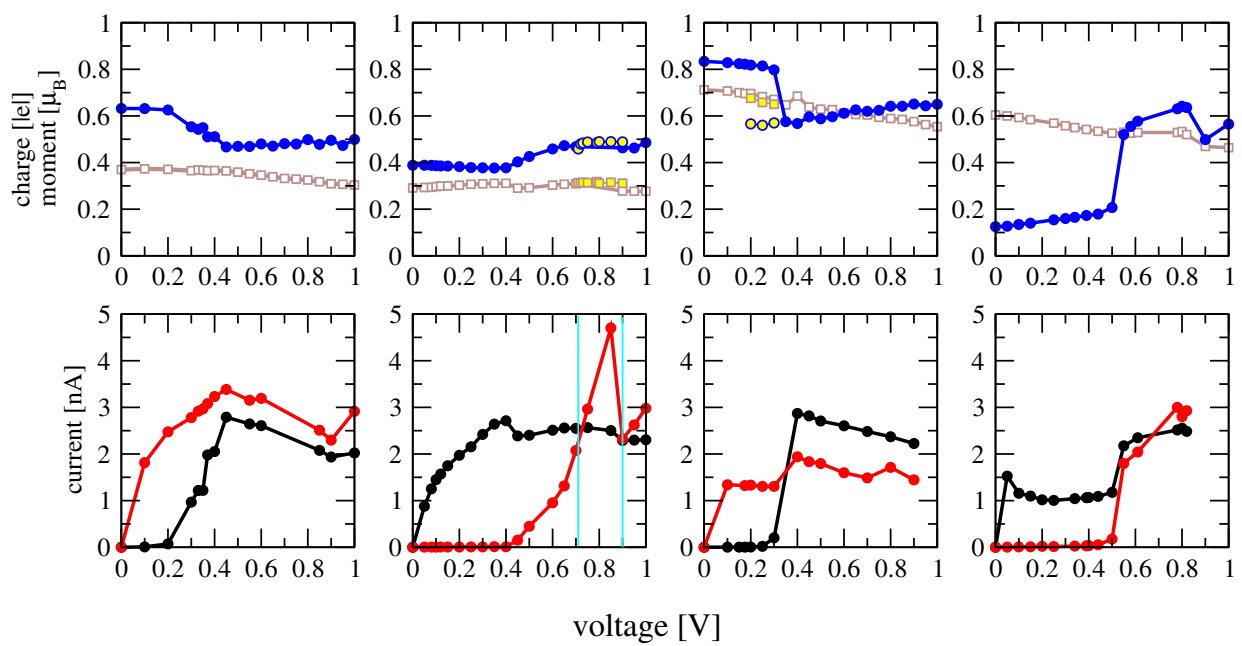

FIG. 7: (color online) Upper panels: net charge (brown squares) and magnetic moment of the molecule (blue circles) as a function of voltage. Lower panels: current versus voltage curves. The four studied cases are ordered from left to right as in figure 6.

In the HS solution the transmission peak related to the HOMO spin-down orbital enters the voltage window near 
$V \sim 0.05 \mathrm{~V}$. This causes a rapid rise of the current for this spin (see figure 7 ). The spin-down component of the current dominates the voltage range from 0.0 to $0.4 \mathrm{~V}$, giving rise to a spin filtering behavior. A peak related to the HOMO-1 spin-up orbital enters the widening voltage window for $V=0.3-0.4 \mathrm{~V}$, and it is accompanied by a steep rise of the spin-up component of the current in this voltage interval. The depopulation of the HOMO-1 spin-up level after its entrance into the voltage window reduces the total magnetic moment of the system in this voltage range. Throughout the whole voltage region, the LUMO spin-down level glides over the upper boundary of the voltage window without entering it, and does not influence much the electron transport.

In the LS solution, the spin-up HOMO level enters the voltage window in a small $V$ region and determines the current until the spin-down HOMO-1 level enters the voltage window in the range 0.5-0.8 $\mathrm{V}$. In effect, the range of the spin filtering behavior is wider in the case of the LS structure than for the HS state.

For the structures doped with acceptors, the HOMO level sticks to an upper edge of the voltage window for most of the voltage interval. It enters then the voltage window once the HOMO-1 level gets inside it. In this way, a depopulation of the HOMO-1 level is counterbalanced by an increase of the population of the HOMO state and the total net charge of the molecule does not change. At the same threshold voltage the magnetic moment of the system exhibits a stepwise change. From zero voltage until entering the HOMO-1 level into the voltage window the majority current is determined by the spin of the HOMO level and a spin filtering behavior is observed. For $V$ bigger than this threshold voltage the spin filtering is inverted, since then HOMO-1 determines the current.

Despite the differences in the details of the voltage evolution of the system for the studied structures, one can also note some common features. First, The HOMO-2 and HOMO-3 spinorbitals move parallel to HOMO and HOMO-1 levels in the studied voltage range, staying away from the voltage window. Second, the total net charge of the molecular complex varies rather slowly with voltage and it does not reflect the steep changes in other system characteristics. Third, the values of the current for $V=1 \mathrm{~V}$ are of similar order of magnitude, reaching a value of 5 nA. These facts are important for determining the parametric model of the system.

An important aspect of the finite voltage (or temperature) evolution of the system is the possibility of finding several branches of metastable solutions. The multistability can be suspected once a solution shows sudden changes of current, transmission peak positions, magnetic moments, or atomic forces.

In some cases two different solutions can be readily found for a range of voltage values. This is exemplified by the LS structure with acceptors (see third column in figure 7 ) for $V=0.2-0.4 \mathrm{~V}$. The bistability is characterised by a sudden decrease of the magnetic moment of the molecular subunit. Here, the forces acting on the atoms are similar for both branches, indicating the proximity of both solutions. The solutions differ in energy by about 0.05 eV and in the distribution of the Mulliken charges in the molecule. Combining these two branches could lead to a spin-dependent NDR effect based on the bistability of the system, that is, a subsequent decrease of the current at voltages around $0.3 \mathrm{~V}$ might be induced by switching to the branch of the first solution, where the HOMO level is still outside the voltage window, thus reducing the current.

In other cases, potential branches of solutions may be masked by the dominating ones and would be hard to locate by a numerical procedure. This may be the case of the LS solution, where the multistability may be suspected from the nonmonotonuos variation of the LUMO level (see figure 6) and the sharp rise of the spin-down component of the current in the range $V=0.7-0.9 \mathrm{~V}$. The forces acting on the atoms are $0.6 \mathrm{eV} / \AA$, while they are typically $0.2 \mathrm{eV} / \AA$ before this point. A subsequent drop of the current (negative differential resistance, NDR) is observed for voltageinduced changes in the electronic structure, which become crucial at higher voltages ${ }^{40}$. A multistable behavior may be also the reason for the step-wise changes of the transmission peaks, the total magnetic moment and the current, which change in the LS case with the acceptors for $V>0.5 \mathrm{~V}$.

\section{A PARAMETRIC MODEL OF THE JUNCTION}

The dependence of the transmission, the current and the magnetic moment on voltage can be understood with the help of a 3-level model which represents the frontier orbitals of the Cu-dioxolene molecule (see figure 4). The orbital of $b_{2}$ symmetry corresponds to a $d$-type orbital, since it is localized mostly on the $\mathrm{Cu}$ atom and its neighborhood. The orbitals of $b_{1}$ and $a_{2}$ symmetry refer to $p 1$ and $p 2$ orbitals, respectively, since they are dominated mostly by the carbon and oxygen $p$ orbitals. The Hamiltonian includes the following parameters:

- $\varepsilon_{d}, U_{d}$ - orbital energy and the Hubbard repulsion for the $\mathrm{Cu}$-centered $(d)$ orbital,

- $\varepsilon_{p \nu}$ - orbital energy for the dioxolene-centered $(p)$ orbitals, $\nu=1,2$,

- $J_{H \nu}$ - ferromagnetic exchange couplings between $d$ and $p$-type orbitals,

- $U_{Q}$ - constant coupling parameter for the total electronic Coulomb repulsion on the molecule, accounting for the near independence of electron occupations on the voltage 
- $\gamma_{\alpha},(\alpha=d, p)$, describe the coupling of the orbitals with the leads, assumed to be independent of energy

The model analysis was performed using the nonequilibrium Green functions method and a mean-field approximation for the interaction terms. The positions of the energy levels read:

$$
\begin{gathered}
E_{d \sigma}=\varepsilon_{d}+\frac{1}{2} U_{d} q_{d}+U_{Q}\left(q_{d}+q_{p 1}+q_{p 2}-Q_{\mathrm{tot}}\right)+\sigma\left[\frac{1}{2}\left(J_{H 1} m_{p 1}+J_{H 2} m_{p 2}\right)-U_{d} m_{d}\right] \\
E_{p \nu \sigma}=\varepsilon_{p \nu}+\frac{1}{2} U_{p} q_{p \nu}+U_{Q}\left(q_{d}+q_{p 1}+q_{p 2}-Q_{\mathrm{tot}}\right)+\sigma\left(\frac{1}{2} J_{H \nu} m_{p \nu}-U_{p \nu} m_{p \nu}\right), \nu=1,2 .
\end{gathered}
$$

$\sigma= \pm 1$ is the spin index, $q_{d}, q_{p \nu}$ denote the electron occupations of the levels $d, p_{\nu}$ and $m_{d}, m_{p \nu}$ are the corresponding magnetizations. The auxiliary parameter $Q_{\text {tot }}$ is included to help controlling the total occupation of the levels. The values of the electron occupations and the magnetizations were computed using the nonequilibrium Green functions method. The density for spin $\sigma$ is computed from the equation:

$$
\rho_{\sigma}=\int \frac{d \omega}{2 \pi}\left(f_{L} G_{\sigma}^{(+)} \Gamma_{L} G_{\sigma}^{(-)}+f_{R} G_{\sigma}^{(+)} \Gamma_{R} G_{\sigma}^{(-)}\right)
$$

where $f_{L}, f_{R}$ are the Fermi factors for the left and right lead, $\Gamma_{L}, \Gamma_{R}$ are the matrices accounting for the coupling with the left and right leads and $G^{(+)}, G^{(-)}$are the retarded and the advanced Green's functions, respectively. In the present case $\Gamma_{L}=\Gamma_{R}=\Gamma$ and the matrices $\Gamma_{L, R}$ and $G^{( \pm)}$are diagonal ones, i.e.:

$$
\begin{aligned}
\Gamma_{\mu \nu} & =\delta_{\mu \nu} \gamma_{\mu} \\
\left(G_{\sigma}^{( \pm)}\right)_{\mu \nu} & =\delta_{\mu \nu} \frac{1}{\omega-E_{\mu} \pm i \gamma_{\mu \sigma}}, \quad \mu=d, p 1, p 2 .
\end{aligned}
$$

The electron occupations $d, p_{\nu}$ and the corresponding magnetizations $m_{d}, m_{p \nu}$ are then computed as:

$$
q=\sum_{\sigma} \rho_{\sigma}, \quad m=\frac{1}{2} \sum_{\sigma} \sigma \rho_{\sigma},
$$

for each of the levels. The value of the current is computed from the Landauer-Büttiker formula (2) where the transmission reads: $T_{\sigma}=\operatorname{tr}\left(\Gamma_{L} G_{\sigma}^{(+)} \Gamma_{R} G_{\sigma}^{(-)}\right)$.

The choice of the model parameters is made to closely reproduce the positions of the transmission peaks of the four DFT solutions at zero voltage. For the structures with full $C_{2 v}$ symmetry we set $U_{d}$ to $2.5 \mathrm{eV}$, taking into account that the $d$ orbital is limited mostly to $\mathrm{Cu}$ atom and its vicinity. The repulsion on the $p$-orbitals, which extends mostly to the whole dioxolene subunit, is expected to be smaller, hence for $U_{p}$ we take the value of $0.1 . .0 .2 \mathrm{eV}$. To describe the twisted structures we take into account that the frontier orbitals are now mixtures of $d$ and $p$ orbitals (cf. figure 3 ) and we take the values $U_{d}=1.7 \mathrm{eV}$ and $U_{p}=1.0 \mathrm{eV}$, so that in all cases $U_{d}+U_{p} \sim 2.6 .2 .7 \mathrm{eV}$. The values of the orbital energies, $\varepsilon_{d}, \varepsilon_{p \nu}$ are fixed by fine tuning of the positions of the SCF orbital levels at the zero voltage for each case. These values determine mostly the occupation of the 3-level orbital system and in this way describe the effect of doping the junction with the acceptors.

The constant coupling parameter $U_{Q}$ is fixed to a value $3.5 \mathrm{eV}$ in all cases. This value should be high enough to account for the fact that the total electron occupation is only weakly dependent on voltage, as it is observed in our DFT results.

The Hund-type intramolecular exchange parameters were assumed to be negative, to favor the parallel alignment of magnetic moments on the $\mathrm{Cu}$ and dioxolene subunits. With the values of these parameters we match the spin splitting of the $p$-orbital levels seen in the DFT computations. To describe the junction with the symmetric molecule, we take $J_{H 1}=-0.3 \mathrm{eV}$, and $J_{H 2}=-0.1 \mathrm{eV}$. In the remaining cases the values of the exchange coupling are of similar order of magnitude.

Finally, the values of the $\gamma_{\mu}$ parameters were adjusted to match the values of the spin components of the current from the DFT computations. For example, to match the values of the DFT results for the junction with the symmetric molecule, we take $\gamma_{d}=0.5 \times 10^{-5} \mathrm{eV}$ and $\gamma_{p}=0.2 \times 10^{-4} \mathrm{eV}$. We stress that the relatively small values of these parameters result from the large energy gap of the alkane linkers. As a consequence, they depend strongly on the linker's length. With these small values of the coupling constants, the results become also strongly dependent on temperature. For this parameter we chose $k_{B} T=0.025 \mathrm{eV}$, as in the DFT computations.

In figure 8 we show the dependence of the selfconsistent (SCF) energy levels on voltage. In figure 9 we plot the net charge, magnetic moment and current for four sets of the model's parameters. 


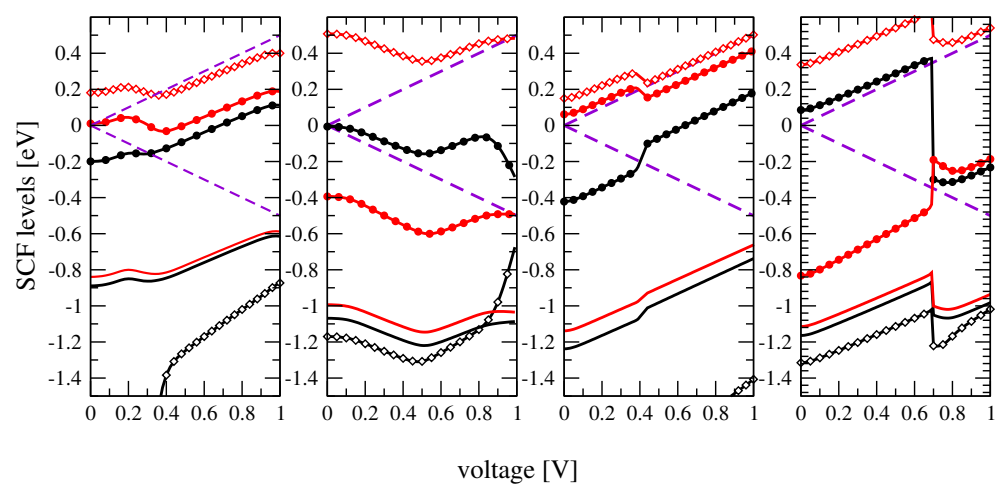

FIG. 8: (color online) The dependence of the energy levels on voltage obtained from the model computations. The black curves represent the levels with spin up, and the red curves the levels with spin down. The broken straight lines show the boundaries of the voltage window. The lines with diamonds is for the $d$-type level and the lines with filled circles are for the higher $p$-type $\left(p_{1}\right)$ levels. The plots have to be compared with the plots of the maxima of the transmission function for the corresponding DFT solutions (from left to right): symmetric without acceptors, twisted without acceptors, symmetric with acceptors and twisted with acceptors.

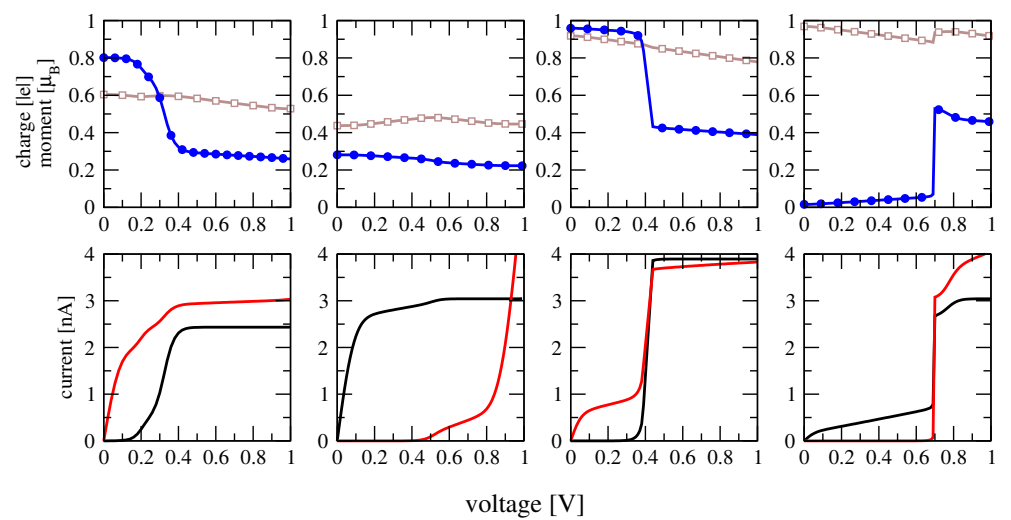

FIG. 9: (color online) Upper row: the dependence of the net charge in the 3-level system (squares) and the total magnetic moment (filled circles) on voltage; lower row: current for spin up (black curve) and spin down (red curve) as obtained from the model computations. The four columns correspond to the cases presented in figure 8.

The dependence of the SCF levels on voltage obtained from the model calculations exhibits the same general features which can be noticed in the dependence of the DFT transmission maxima on voltage, presented in figure 6. First, we see that throughout most of the voltage region, the SCF levels travel along parallel lines. Second, once a peak approaches the voltage window it changes direction and starts to show a tendency to slide over the edge over some voltage range, rather than entering the window instantaneously. Third, the sudden change of direction of the peak approaching the window induces a similar response in peaks far from the window. In general, this coordinated evolution of the SCF levels follows from the tendency of the system to keep the charge of the molecule nearly constant (see figure 9), despite substantial variation of the total magnetic moment and the entrance of different levels into the voltage window. In the present model, the preservation of the charge it is ensured by assuming a strong enough value of the parameter $V_{Q}$, which, depending on the change of the total occupation, shifts all the SCF levels by the same amount. The physical reason for such behavior in the DFT results can be traced back to the long range Coulomb interactions, including the repulsion between charge fluctuations on the molecule and on the linkers as well as the interaction with the image charge in the leads. In the parametric model, including the constant coupling parameter $V_{Q}$ takes indirectly into account these effects, even if we do not include interactions with the electrons from the leads or the linkers explicitly into the model's Hamiltonian. Other common features of the DFT results and the model's ones is the appearance of jumps of the total magnetization for voltage values for which the SCF levels approach the voltage window (see figure 9). We also note that the current-voltage dependence of the DFT computation is rather well reproduced by the present model. 


\section{SUMMARY AND FINAL REMARKS}

We have studied a simplified model of a molecular junction (copper-dioxolene between gold (111) electrodes), that exhibits valence tautomerism and spin crossover properties. The metalorganic subunit was linked to the leads with alkanethiole linkers. The linkers effectively protect the magnetic and tautomeric features of the molecule from perturbations coming from the electrons of the metal. The spectral and the transport properties of the junction can be unambiguously related to the energy levels of the metalorganic subunit. From the GGA+U results we found two possible solutions. The first one was a symmetric configuration, with the magnetic moment located mostly on the copper atom. The other solution describes a twisted molecule, with the magnetic moment reduced and the electronic charge shifted towards the dioxolene ligand. The analysis of the bond lengths and the Mulliken charges allowed us to assign these solutions to different valence tautomers.

The transport properties of both configurations were computed with a combination of the DFT+U approach and the non-equilibrium Green's function method. The current at $V=1 \mathrm{~V}$ is of a similar order of magnitude as that of an alkanethiole chain of comparable length, ${ }^{41}$ but the insertion of the Cu-dioxolene molecule in the chain significantly enriches the current-voltage $(I-V)$ characteristics. The transmission functions exhibit a series of sharp peaks near the Fermi level that can be clearly linked to the eigenstates of the metalorganic complex. The positions of the peaks are found to vary with the voltage in a coordinated manner. The $I-V$ curves of the two studied conformations clearly differ, but in both cases the junction exhibits a strong spin filtering effect. The spin-filtering is due to the spin splitting of the molecular levels by the Coulomb and the exchange interactions as well as by the proximity of spin-polarized levels to the Fermi energy of the junction. This latter property is related to the incomplete charge transfer from the metalorganic subunit to the gold leads. We showed that the charge transfer depends only weakly on the applied voltage, which explains the persistence of the spin-filtering effect for a finite voltage range.

We showed that by doping the junction with acceptor ions it is possible to introduce some features in the transport characteristics. The presence of acceptors significantly decreases the electron occupation of the $\mathrm{Cu}-\mathrm{dioxolene}$ subnit, by about 0.3 e for both studied conformations. This change resulted in an upward shift of the HOMO level of the molecule above the Fermi level of the junction. We also found a sudden inversion of the spin filtering behavior for certain voltages. Moreover, we found indications of a multistable behavior of the system for a finite voltage range, that can lead to NDR behavior. We used the results of the DFT approach to determine the parameters of an effective parametric model. The parametric model's analysis also reveals the existence of jumps of the magnetization of the molecule and the current of the junctions for certain voltage values as well as a multistability of the solutions. The model calculations reproduce semiquantitatively the main features of the DFT results. The rich behavior of the model, despite its simplicity, shows that it may be used as a universal tool to study a potentially wide class of junctions with metalorganic molecules exhibiting SCO transitions. The model's simple mathematical structure is advantageous for an improved treatment of electron correlations in such systems. Work in this latter direction is in progress.

\section{Acknowledgments}

We thank Bogdan Bułka for critical reading of the manuscript and helpful advice. This work has been supported by the National Science Centre under the contract DEC-2012/07/B/ST3/03412. Our computations were performed at the Poznań's Supercomputing and Networking Center. V.M.G.-S. thanks the Spanish Ministerio de Economía, Industria y Competitividad for funding through the project FIS2015-63918-R.

1 A. Bousseksou, G. Molnár, L. Salmon, and W. Nicolazzi, Chem. Soc. Rev. 40, 3313 (2011).

2 S. Sanvito, Chem. Soc. Rev. 40, 3336 (2011).

3 C. Lefter, V. Davesne, L. Salmon, G. Molnár, P. Demont, A. Rotaru, and A. Bousseksou, Magnetochemistry 2, 18 (2016).

${ }^{4}$ P. Gütlich, Y. Garcia, H. A. Goodwin, K. S. Murray, J. Shu, R. J. Hemley, H. K. Mao, N. A. Young, F. Varret, O. Kahn,

D. R. Smith, K. Wieghardt, M. Zehnder, and A. D. Zuberbühler, Chem. Soc. Rev. 29, 419 (2000).

5 P. Gamez, J. S. Costa, M. Quesada, and G. Aromí, Dalt. Trans., 7845 (2009).

${ }^{6}$ P. Gütlich, A. B. Gaspar, and Y. Garcia, Beilstein J. Org. Chem. 9, 342 (2013).

7 E. Ruiz, Phys. Chem. Chem. Phys. 16, 14 (2014).

8 R. González-Prieto, B. Fleury, F. Schramm, G. Zoppellaro, R. Chandrasekar, O. Fuhr, S. Lebedkin, M. Kappes, and M. Ruben, Dalt. Trans. 40, 7564 (2011).

9 J. Dugay, M. Aarts, M. Giménez-Marqués, T. Kozlova, H. W. Zandbergen, E. Coronado, and H. S. J. van der Zant, Nano Lett. 17, 186 (2017).

10 P. Gütlich, V. Ksenofontov, and A. Gaspar, Coord. Chem. Rev. 249, 1811 (2005). 
11 G. A. Craig, J. S. Costa, O. Roubeau, S. J. Teat, H. J. Shepherd, M. Lopes, G. Molnár, A. Bousseksou, and G. Aromí, Dalt. Trans. 43, 729 (2014).

12 S. Decurtins, P. Gutlich, K. M. Hasselbach, A. Hauser, and H. Spiering, Inorg. Chem. 24, 2174 (1985).

13 A. Hauser, Chem. Phys. Lett. 124, 543 (1986

14 B. Li, R.-J. Wei, J. Tao, R.-B. Huang, L.-S. Zheng, and Z. Zheng, J. Am. Chem. Soc. 132, 1558 (2010).

15 E. Coronado, M. Giménez-Marqués, G. Mínguez Espallargas, F. Rey, and I. J. Vitórica-Yrezábal, J. Am. Chem. Soc. 135, 15986 (2013)

16 J. Sánchez-Costa, S. Rodríguez-Jiménez, G. A. Craig, B. Barth, C. M. Beavers, S. J. Teat, and G. Aromí, J. Am. Chem. Soc. 136, 3869 (2014).

17 F. Prins, M. Monrabal-Capilla, E. A. Osorio, E. Coronado, and H. S. J. van der Zant, Adv. Mater. 23, 1545 (2011).

18 C. Lefter, S. Rat, J. S. Costa, M. D. Manrique-Juárez, C. M. Quintero, L. Salmon, I. Séguy, T. Leichle, L. Nicu, P. Demont, A. Rotaru, G. Molnár, and A. Bousseksou, Adv. Mater. 28, 7508 (2016).

19 M. Bernien, H. Naggert, L. M. Arruda, L. Kipgen, F. Nickel, J. Miguel, C. F. Hermanns, A. Krüger, D. Krüger, E. Schierle, E. Weschke, F. Tuczek, and W. Kuch, ACS Nano 9, 8960 (2015).

20 K. Park, S. Barraza-Lopez, V. M. García-Suárez, and J. Ferrer, Phys. Rev. B 81125447 (2010).

21 N. Baadji and S. Sanvito, Phys. Rev. Lett. 108, 217201 (2012).

22 T. Miyamachi, M. Gruber, V. Davesne, M. Bowen, S. Boukari, L. Joly, F. Scheurer, G. Rogez, T. K. Yamada, P. Ohresser, E. Beaurepaire, and W. Wulfhekel, Nature Commun., 3, 938 (2012).

${ }^{23}$ E. Cremades, C. D. Pemmaraju, S. Sanvito, and E. Ruiz, Nanoscale 5, 4751 (2013).

24 R. Ferradás, V. M. García-Suárez, and J. Ferrer, J. Phys. Cond. Matter 25, 325501 (2013).

25 A. Droghetti, D. Alfé, and S. Sanvito, J. Chem. Phys. 137, 124303 (2012).

${ }^{26}$ V. Meded, A. Bagrets, K. Fink, and R. Chandrasekar, M. Ruben, F. Evers, A. Bernand-Mantel, J. S. Seldenthuis, A. Beukman, and H. S. J. van der Zant, Phys. Rev. B 83, 245415 (2011).

27 A. D. Becke, J. Chem. Phys. 98, 5648 (1993).

28 C. Lee, W. Yang and R. G. Parr, Phys. Rev. B 37785 (1988).

29 C. Toher and S. Sanvito, Phys. Rev. Lett. 99, 056801 (2007).

30 V. I. Anisimov, J. Zaanen, and O. K. Andersen, Phys. Rev. B 44 (1991) 943-954.

31 M. Cococcioni and S. de Gironcoli, Phys. Rev. B 71, 035105 (2005).

32 T. Kostyrko and T. Ślusarski, Appl. Surf. Sci. 373, 19 (2016).

33 The figures with structures of $\mathrm{Cu}$-dioxolene were made using Jmol: an open-source Java viewer for chemical structures in 3D. http://www.jmol.org/.

34 P. Ordejon, E. Artacho and J. M. Soler, Phys. Rev. B 53, R10441 (1996); J. M. Soler, E. Artacho, J. D. Gale, A. García, J. Junquera, P. Ordejón, and D. Sánchez-Portal, J. Phys. Condens. Matter 14, 2745 (2002).; N. Papior et al. Computer Physics Communications Volume 212, March 2017, Pages 8-24

35 J. P. Perdew, K. Burke, M. Erzenhof, Phys. Rev. Lett. 77, 3865 (1996).

36 P. Rivero, C. Loschen, I. de P. R. Moreira, and F. Illas, J. Comput. Chem. 30, 2316 (2009).

37 N. Troullier and J. L. Martins, Phys. Rev. B 43, 1993 (1991).

38 J. Ferrer, C. J. Lambert, V. M. García-Suárez, D. Z. Manrique, D. Visontai, L. Oroszlany, R. Rodríguez-Ferradáas, I. Grace, S. W. D. Bailey, K. Gillemot, H. Sadeghi and L. A. Algharagholy, New J. Phys. 16, 093029 (2014).

39 XCrySDen, see: A. Kokalj, Comp. Mater. Sc. 28, 155 (2003)

40 V. M. García-Suárez and C. J. Lambert, Nanotechnology 19, 455203 (2008).

41 B. Xu and N. J. Tao, Science 301, 1221 (2003). 